Gesnerus 69/2 (2012) 247-271

\title{
Des prêtres-médecins en Russie
}

Hélène Berlan, Dominique Triaire

\section{Summary}

Jean Pierre Frank offers in the early nineteenth century a revolution in medical Russian Empire. Indeed, Russia is in an emergency situation where the lack of practitioners is obvious. The imperial project is inspired by past practices in some European countries. Frank fits these transfers and implements a unique model where the priest-doctor stands out as the solution to overcome the lack of medicalization of the Empire. Even if the attempt was a failure, it remains that the proposals were part of Frank in both an ancient tradition that priests and physicians providing care for souls and bodies, but also showed that called his wishes the advent of "public health" in this country disinherited.

Keywords: priest-physician, Russian Empire, transfer of experiences

\section{Sommaire}

Jean Pierre Frank propose au début du XIX $\mathrm{X}^{\mathrm{e}}$ siècle une véritable révolution médicale à l'Empire russe. En effet, la Russie se trouve dans une situation d'urgence où le manque de praticiens est patent. Le projet impérial s'inspire de pratiques déjà éprouvées dans certains pays d'Europe. Frank adapte ces transferts et met en place un modèle original où le prêtre-médecin s'impose comme la solution pour pallier l'absence de médicalisation de l'Empire. Même si la tentative a été un échec, il n'en demeure pas moins que les propositions de Franck s'inscrivaient à la fois dans une tradition millénaire celle des prêtres-médecins apportant soins aux âmes et aux corps, mais montraient aussi qu'il appelait de ses vœux l'avènement de la «santé publique» dans cette contrée déshéritée.

Dr Hélène Berlan, Université Montpellier III, helene.berlan@univ-montp3.fr. Prof. Dr Dominique Triaire, Université Montpellier III, dominique.triaire@univ-montp3.fr. 


\section{La structure médicale de l'Empire russe: l'urgence de l'action Le «désert médical» de l’Empire}

Le nom de Jean-Pierre Frank (1745-1821) n'est pas inconnu dans l'histoire de la médecine. Il naît à Rodalben, en Rhénanie-Palatinat, puis poursuit ses études à Heidelberg et Strasbourg. Là, il évolue dans un milieu universitaire ouvert sur l'ensemble de l'Europe ${ }^{1}$ et plus spécifiquement le Saint Empire: les étudiants s'expriment en allemand et, surtout, il règne une émulation entretenue par les relations que l'institution universitaire a tissées avec les Académies et Sociétés Savantes. ${ }^{2}$ Jean-Pierre Frank soutient sa thèse de médecine en 1766 à Heidelberg et exerce ensuite à Rastatt et à Baden; puis il devient professeur de médecine à l'Université de Göttingen en 1784, à celle de Pavie, un an plus tard. En 1795, il est nommé professeur de médecine pratique à l'Université de Vienne et directeur de l'hôpital général.

Le 17 octobre 1803, il prononce à l'Université de Vienne «un discours sur l'exercice du sacerdoce et de la médecine réunis en une seule personne, [...] pour l'ouverture des écoles» ${ }^{3}$ dans lequel il retrace depuis l'Antiquité l'histoire des prêtres-médecins. En butte à l'hostilité de ses confrères et de ses supérieurs, il accepte la proposition d'enseigner à l'Université de Vilna où il arrive le 4 octobre 1804. Il y séjourne jusqu'au 23 juillet 1805. Répondant à l'appel de l'empereur Alexandre ${ }^{\mathrm{er}}$, il prend le chemin de Saint-Pétersbourg où il arrive le 15 août suivant. Il y est nommé directeur général de l'Académie médico-chirurgicale, mais aussi médecin personnel de l'empereur.

La Russie souffrait cruellement du manque de médecins autant dans ses armées que dans la population civile. Frank signale qu'un émigré français, le comte de Wargemont, ${ }^{4}$ chambellan de l'empereur de Russie, avait proposé en 1801 de joindre «forcément l'étude de la médecine et de la chirurgie à celle de la théologie, de sorte que tout homme curé ou tout vicaire de village pourroit et seroit forcé d'y porter ces secours». ${ }^{5}$ Le discours prononcé par Frank en 1803 apportait au projet de Wargemont un fondement historique; il n'est donc pas étonnant que Frank fût sollicité en ce sens dès son arrivée dans la capitale russe.

1 Strasbourg devient dès 1793 une des trois facultés de médecine avec Paris et Montpellier; toutes les autres à cette date ont été supprimées.

2 Voir Livet.

3 Mémoires biographiques, ch. XXIX. Le «discours» fut édité: «Akademische Rede über die Priesterärzte», Taschenbuch 1804, III-LX.

4 Il semble qu'il s'agisse d'Albert-François Le Fournier, comte de Wargemont, né en 1754, qui émigra en Russie en 1792. Revenu en France en 1803, il repartit pour Saint-Pétersbourg où il mourut sans descendance.

5 Frank 1817, vol. VI/2, 438. Nous remercions M. Lorenz Frischknecht de l'aide apportée pour accéder à cet ouvrage. 
Plus surprenant est le nom de l'intercesseur choisi: Adam Jerzy Czartoryski (1770-1861), ami personnel du tsar, curateur de l'Université de Vilna, en charge des Affaires étrangères de l'Empire, s'était impliqué personnellement dans l'invitation de Frank en Lituanie et aurait dû être chargé de lui demander de réfléchir à la formation médicale des ecclésiastiques. Il n'en fut rien et pour deux raisons: Czartoryski avait peu apprécié que Frank eût quitté Vilna; en outre, à la fin de 1805, date probable de la lettre rapportée ci-dessous, il est en semi-disgrâce à la suite d'Austerlitz. C'est donc le comte Séverin Potocki (1762-1829), ${ }^{6}$ sénateur de Russie, curateur de l'Université de Kharkov, de sa fondation jusqu'en 1817, qui prend contact avec Frank. Les deux hommes avaient fait connaissance lors d'un dîner organisé à l'Université de Vilna, peu avant le départ de Frank pour Saint-Pétersbourg. ${ }^{7}$

Le projet impérial pour lequel Frank est sollicité est ambitieux. C'est un moyen de répondre à une situation d'extrême urgence: former un personnel médical de qualité pour un immense empire composé à $95 \%$ de territoires ruraux. ${ }^{8}$ Or, dans cet espace «infini», le constat d'une sous-médicalisation est patent. Selon les travaux de Ludovic Debono, on ne compte au début du XIX ${ }^{\mathrm{e}}$ siècle que 1500 médecins pour tout l'Empire, ce qui représente un praticien pour 24000 Russes. Mais ce chiffre est trompeur et doit être nuancé, car de profondes inégalités existent. En effet, la répartition du personnel médical indique que deux tiers des hommes de l'art résident dans les zones urbaines, le dernier tiers étant des médecins militaires qui pratiquent au sein de leur institution d'origine. Ce sont donc les villes qui bénéficient exclusivement d'une assistance médicale et, là encore, des disparités apparaissent, car la clientèle de ces médecins n'est composée que de riches marchands et de l'élite aristocratique. De ce fait, comme l'indique Debono, «la quasi totalité de la population vit sans assistance médicale». ${ }^{9}$

Au-delà de ces indicateurs qui témoignent du caractère incommensurable de la tâche, il faut immédiatement souligner que traditionnellement l'avènement d'un tsar, jeune de surcroît (24 ans en 1801), suscite attentes et espérances pour une réorganisation en profondeur de l'Empire.

\footnotetext{
6 Frère de Jean, l'auteur du Manuscrit trouvé à Saragosse.

7 Mémoires biographiques, ch. XLVI.

8 La Russie d'Europe est divisée en 50 provinces, chacune d'entre elles représente environ un cinquième de la France (Debono 1997).

9 Debono 1997, 99.
} 


\section{Les premières réformes et le rôle central de l'Académie médico-chirurgicale}

Le discours d'octobre 1803 de Frank et en raison de l'aura intellectuelle de celui-ci dans l'Europe du début du XIX ${ }^{\mathrm{e}}$ siècle en tant que penseur des politiques générales de «santé publique» a retenu l'attention du prince et c'est donc, assez naturellement, que Frank est appelé à la cour où il devient médecin personnel du souverain et directeur de l'Académie médico-chirurgicale à partir de juillet $1805 .{ }^{10}$ Alexandre $\mathrm{I}^{\text {er }}$ a cependant initié, quelques années avant l'arrivée de Frank, un train de réformes. En effet, dès le début de son règne, l'enseignement supérieur connaît une expansion remarquable et quatre universités sont créées et vont s'ajouter à celle de $\mathrm{Moscou}^{11}$ avec, à chaque fois, une faculté de médecine. Dans cette perspective de modernisation, le tsar a également entrepris une réforme du système de santé et, ce, dès 1802. À cette date, l'ancien Collège Médical est remplacé par un bureau médical impérial, placé sous la direction du ministère de l'Intérieur nouvellement créé. ${ }^{12}$ Ce bureau médical est divisé en deux structures distinctes: la première, le Conseil Médical, devient la plus haute autorité de l'Empire pour les questions de santé, elle a en effet la charge de la formation des personnels et de la politique médicale en général; la seconde, le Département Médical, est en relations directes avec le terrain, dirige l'activité médicale de l'Empire, centralise les informations et envoie les ordres. ${ }^{13}$ Ces deux institutions constituent la clef de voûte du système de santé russe et commandent à l'ensemble des autres organismes: les facultés de médecine, les médecins de cour et l'Académie médico-chirurgicale. Enfin, lors de l'arrivée de Frank en Russie, une dernière réforme a vu le jour en 1805: elle consiste en la séparation entre la médecine militaire et la médecine civile, celle-là passant sous le contrôle du ministère de la guerre. ${ }^{14}$

Il existe une raison peut-être plus spécifique au choix de Frank pour présider aux destinées de l'Académie médico-chirurgicale: il s'agit du fait qu'il est allemand. En effet, même si c'est à Pierre le Grand que l'on doit les premières réformes universitaires ${ }^{15}$ et à Lomonossov, par la suite, l'organisa-

10 Voir Frioux/Fournier/Chauveau 2011. Jean-Pierre Frank exerce les fonctions de directeur de l'Académie médico-chirurgicale de Saint-Pétersbourg jusqu'en 1808.

11 On compte l'université de Vilnius, prise aux Polonais, et celle de Dorpat, prise aux Suédois. Auxquelles s'ajoutent des créations: Kazan et Kharkhov en 1804 (Debono 1997, 143, 89, 100, $51,183)$.

12 Debono 1997, 143, 191. Ce Collège Médical, organisme d’État, était la faculté de médecine de Moscou.

13 Debono 1997, 143.

14 Debono 1997, 191.

15 Debono 1997, 89. Le premier hôpital moderne de la Russie, le Gofspital, est créé en 1707 à Moscou. 
tion de l'enseignement de la médecine ${ }^{16}$ le système présentait deux carences de taille. La première était qu'il n'existait avant cette réforme aucune formation médicale spécifique dans l'Empire même. De ce fait, les étudiants qui souhaitaient embrasser la carrière devaient se rendre à l'étranger.La seconde était que même si Catherine II avait accordé au Collège Médical, organisme d'État, le droit de délivrer le titre doctoral (ce qui était une amélioration par rapport à la situation précédente), ce Collège était composé d'enseignants allemands qui répugnaient à «accorder le titre [de docteur] à ces Russes mal dégrossis». Après l'intervention de la tsarine, le premier doctorat n'est délivré qu'en 1768 et il faudra attendre 1783 pour que de nouveaux docteurs soient diplômés et ce n'est qu'en 1791 que l'Université de Moscou reçoit le droit de conférer le titre de sa propre autorité. ${ }^{17} \mathrm{Au}$-delà de ces éléments qui témoignent des pesanteurs du système, de l'incapacité de l'Empire, puis des divers freins à assurer la formation de ses propres médecins, on remarque qu'il existe en Russie une tradition bien établie de recours à des personnels étrangers compétents, en particulier allemands et plus spécifiquement dans le domaine de la médecine. C'est donc peut-être assez naturellement que Frank, Allemand, médecin et de plus auteur d'un projet audacieux, a pu être appelé auprès d'Alexandre I ${ }^{\text {er }}$ pour finaliser la réforme. De plus, l'Académie médico-chirurgicale est un établissement de prestige: il est la dernière école de médecine du XVIII ${ }^{e}$ siècle $^{18}$ et c'est aussi la meilleure. Ainsi, la nomination de Frank à la tête de cette fameuse institution est également la marque de la confiance que le tsar lui accorde et celle des espoirs qu'il place en lui.

Après avoir évoqué le contexte dans lequel Frank arrive à Saint-Pétersbourg en août 1805 et les récentes transformations institutionnelles dans le domaine médical universitaire en ce début de $\mathrm{XIX}^{\mathrm{e}}$ siècle, il faut envisager en quoi le projet proposé par le tsar Alexandre $\mathrm{I}^{\text {er }}$ revêt un caractère inédit et comment il permet avec Frank, chargé de lui donner corps, de répondre à l'urgence de la situation médicale dans l'Empire.

16 Lomonossov crée la première section médicale de Russie à Moscou en 1755. La première faculté de médecine est créée en 1764. L'enseignement y est exclusivement théorique (Debono 1997, 89, 51, 143, 74).

17 Debono 1997, 89, 143, 221, 74.

18 Debono 1997; celle de Moscou, fondée en 1799 par Paul I ${ }^{\text {er }}$, ayant été intégrée en 1802 à la faculté de médecine de Moscou pour éviter d'entrer en concurrence avec elle. 


\section{L'Empire à l'heure européenne: les transferts d'expériences et la naissance d'un modèle original}

\section{L'ouverture et les emprunts faits à la France et aux Cantons suisses}

Dans un premier temps, l'oukase présente la synthèse de différentes expériences européennes. L'écrit impérial montre qu'Alexandre ${ }^{\text {er }}$ s'est approprié une tradition européenne qui remonte parfois au milieu du XVII ${ }^{\mathrm{e}}$ siècle. Celle-ci n'a pu être connue du tsar que par le cosmopolitisme de sa cour impériale, attesté par la présence de nombreux nobles émigrés français, comme en témoignent les fonctions occupées par le comte de Wargemont, auteur de la proposition de 1801, ou encore l'éducation francophone du jeune souverain; Alexandre $\mathrm{I}^{\mathrm{er}}$ a en effet été éduqué à la française par Frédéric César de la Harpe ${ }^{19}$ qui lui a inculqué le culte des Lumières et, plus largement, de la culture française. ${ }^{20}$ L'histoire familiale du souverain montre qu'il a aussi des liens avec le Saint-Empire; il est le fils de Dorothée de Wurtemberg et il a épousé en 1793 Louise-Sophie-Augusta de Bade. C'est donc un souverain pétri de culture franco-germanique qui accède au trône. De plus, dès son avènement, il constitue un cabinet de personnes adhérant aux idées libérales; ${ }^{21}$ les thèmes développés seront l'abolition du servage ou l'éventualité de doter le pays d'une constitution. Tous ces éléments révèlent que le tsar a grandi et évolué dans un milieu résolument ouvert sur l'Europe de l'Ouest, ses expériences du despotisme éclairé et ses récentes transformations.

Le tsar souhaite faire bénéficier l'Empire des avancées qui, dans le domaine médical, ont déjà porté leurs fruits ailleurs en Europe. Il s'agit pour lui d'importer par degrés un modèle dans le domaine de la «santé publique». Ainsi, l'oukase s'inspire de certaines spécificités françaises. Le souci de composer «une instruction abregée mais claire de la manière de traiter les

19 Originaire de la ville de Lausanne et après des études de droit, Catherine II lui confie l'éducation de ses deux petits-fils, Alexandre et Constantin.

20 Invité en Russie, François-Adrien Boieldieu devient le compositeur officiel de la cour de 1804 à 1810 .

21 Maya Goubina cite les deux références suivantes:Victor Léontovitch, Histoire du libéralisme en Russie (Paris 1986): «L'époque d'Alexandre I ${ }^{\text {er }}$ fut avant tout celle de la diffusion des idées libérales en Russie, une époque qui vit mûrir la conscience libérale des hautes sphères de la société, où évoluèrent les représentants les plus importants de ce courant politique» (Goubina 2008, 56). Selon Jean Bonamour, Alexandre permit, de nouveau, le fonctionnement des imprimeries privées et supprima l'interdiction d'importer des livres étrangers (Bonamour 1965, 62). En juillet 1804, fut publiée la Loi de censure qui est considérée comme la plus libérale parmi toutes les lois de censure russes. De nouvelles universités furent fondées. «Une réforme très libérale» des universités fut promulguée en 1804, elle «leur donnait une plus grande autonomie et laissait dans le système d'enseignement une plus grande place à l'initiative des étudiants». 
maladies les plus ordinaires aux habitans de la campagne, en y joing[n]ant la déscription des rémèdes» ${ }^{22}$ ressemble à bien des égards à l'initiative prise par Louis XIV en 1706 qui, «touché de compassion pour les pauvres malades de la campagne qui périssaient faute de secours», institue la distribution de boîtes de remèdes qui seront préparées par le médecin Jean-Adrien Helvétius et envoyées chaque année dans les généralités à destination des populations rurales. ${ }^{23} \mathrm{En}$ imitant la démarche de Louis XIV, le tsar ne fait qu'accomplir son métier de prince, c'est en effet un devoir pour le monarque de soulager les souffrances de son peuple; la lettre du comte Potocki, introduction à l'oukase, précise d'ailleurs que le tsar désire «procurer des moyens de secours aux habitans des campagnes dans leurs maladies». ${ }^{24} \mathrm{Si}$ l'initiative française a davantage relevé de l'effet d'annonce, car les boîtes de remèdes sans cesse demandées n'étaient pas en nombre suffisant, on note que la préparation de la boîte était scrupuleuse et que l'édition d'un livret spécifique l'accompagnait. Celui-ci, élaboré à partir des pharmacopées savantes, celles de Moïse Charas ou de Nicolas Lemery, était un véritable ouvrage de vulgarisation scientifique ${ }^{25}$ qui présentait les différentes drogues et les pathologies pour lesquelles on devait les utiliser. Chaque boîte était composée, entre autres, de poudres, pilules, élixir, boules médicamenteuses; on y trouvait aussi deux onces de thériaque, une once de quinquina ou de l'onguent de Nuremberg utilisé pour les «playes, panaris, ulcères, brûlures ou engelures». ${ }^{26}$ En Russie, cette tâche incombera au Collège de Médecine.

Par ailleurs, les campagnes françaises étaient dépourvues de médecins, comme en Russie; la distribution des médicaments devait être confiée en priorité aux ecclésiastiques, puis aux personnes charitables. Ainsi, les ouvrages d'automédication que l'on trouve en France à la fin du XVII ${ }^{\mathrm{e}}$ siècle et qui ont connu un énorme succès, sont adressés en priorité aux ecclésiastiques, comme l'indique l'épître dédicatoire des Remèdes charitables de Madame Fouquet: ${ }^{27}$

A Messieurs les prestres, diacres \& Clercs des Séminaires, \& autres Ecclesiastiques destinés aux fonctions curiales [...] Recevés-le [le recueil] s'il vous plait, Messieurs avec le même cœur que je vous le présente. Travaillés hardiment à la composition des Receptes que vous y verres. Suivés fidelement la méthode de leur préparation, comme nous vous l'y donnons. Conseillés en charitablement l'usage à ceux qui seront dans la necessité de s'en servir. [...]

22 Voir ci-dessous, document II, p. 260.

23 Trépardoux 1996, Lafont 2002.

24 Voir ci-dessous, document I, p.259.

25 Lafont 2002, 213.

26 Arch. dép. Côte-d'Or, C 364. Boîtes de remèdes sous le règne de Louis XV.

27 Lafont 2002, 215-216. 
Et soyez aussi bien Médecins de leurs corps, que vous l'êtes de leurs ames. Cette occupation n'est pas indigne de vôtre sacré Ministère, puisqu'elle est fondée sur l'exemple de Jesus-Christ, \& de ses Apôtres, de qui vous êtes de parfaits Imitateurs.

Dans la seconde moitié du XVIII ${ }^{\mathrm{e}}$ siècle, dans l'introduction de son célèbre Avis au peuple sur sa santé, Samuel-Auguste-David Tissot s'adresse également au personnel religieux:

[...] le titre d'avis au peuple n'est point l'effet d'une illusion qui me persuade que ce livre va devenir une pièce de ménage dans la maison de chaque paysan: les dix-neuf-vingtièmes ne sauront sans doute jamais qu'il existe; plusieurs ne sauroient pas le lire; un plus grand nombre, quelque simple qu'il soit, ne le comprendroit pas. Mais je le destine aux personnes intelligentes, et qui, par une espèce de vocation de la providence sont appelées à aider de leurs conseils tout le peuple qui les environne. L'on sent aisément que j'ai en vue, premièrement messieurs les pasteurs: il n'y a point de village, de hameau, de maison foraine dans tout le pays, qui n'ai droit à la bénéficience d'un d'entre eux $[\ldots]$.

\section{La solution du prêtre-médecin}

On le voit, dans l'Europe de la fin du XVIII ${ }^{\mathrm{e}}$ siècle, le problème de l'accès aux soins pour les populations rurales s'était posé et même si les États avaient inégalement répondu face à l'ampleur de la tâche, ces expériences étaient transposables dans la Russie du début du XIX ${ }^{\mathrm{e}}$ siècle. Ainsi l'oukase propose que «le moyen le plus convenable d'atteindre ce but [fournir des secours aux habitants des campagnes], serait de confier ces soins aux prêtres des villages en leur procurant toute la facilité à pouvoir réunir avec les connaissances conformes à leur vocation celles de la médecine». ${ }^{28}$

Le prêtre charitable (et en outre médecin en Russie) n'est pas le seul emprunt qu'Alexandre I ${ }^{\text {er }}$ fait à l'Europe: conscient des ravages que l'absence de personnel compétent fait courir à son Empire, il souhaite, comme en France dans la seconde moitié du XVIII ${ }^{\mathrm{e}}$ siècle, lutter contre le charlatanisme qui sévissait essentiellement dans les campagnes et que la Société Royale de Médecine de Paris avait tenté d'éradiquer. ${ }^{29}$ L'oukase précise qu' «il est connû

28 Voir ci-dessous, document II, p. 260.

29 La Société Royale de Médecine est créée le 29 avril 1776 par arrêt du Conseil du roi, sous le nom de Commission de médecine (Lettres patentes du 29 avril 1776), pour tenir une correspondance avec les médecins de provinces à propos des maladies épidémiques et épizootiques. Elle est réunie en 1778 à la «Commission pour l'examen des remèdes secrets et des eaux minérales», et devient Société Royale de médecine (SRM) par lettres patentes du 20 août 1778. 
qu'il n'y a point des médecins dans les villages, et que les accidens les moins graves y déviennent mortels par l'ignorance, par des prejugés invétérés et par la manière de traiter les maladies». ${ }^{30}$ Effectivement dans les campagnes russes, le médecin de la ville est un quasi inconnu et il est souvent regardé comme un étranger. Les habitants n'ont pas confiance dans les traitements qui leur sont délivrés; ils préfèrent recourir aux rebouteux et guérisseurs qu'ils connaissent bien et qui sont versés soit dans l'art des simples comme la znakharka, vieille femme résidant au village ou itinérante, le koudesnik qui soigne à l'aide de mystérieuses formules magiques ou encore le kostopraz, rebouteux adroit pour soigner les traumatismes. ${ }^{31}$ On a donc ici une situation assez proche de celle que l'on pouvait rencontrer dans les campagnes françaises. L'entreprise est immense, car elle va inéluctablement se heurter aux archaïsmes et aux pesanteurs, mais aussi aux traditions de cette société rurale russe. Néanmoins la nouveauté des prêtres-médecins en Russie, par rapport aux expériences européennes qui ont précédé, est la mise en place d'une véritable institution, sous l'autorité du tsar, à la tête du Saint Synode, avec création d'un double cursus universitaire, d'abord en théologie, puis en médecine. Partout ailleurs en Europe, le personnel religieux n'était qu'un auxiliaire, un relais du pouvoir accomplissant aussi un devoir charitable et surtout instruit a minima pour dispenser les secours sans que ce dispositif soit légalement encadré. Ceci était du reste interdit au sein de l'Église romaine et à de nombreuses reprises au cours de la période médiévale, des conciles avaient insisté sur ce point. ${ }^{32}$ De nombreuses autres objections avaient été formulées, notamment le fait que les prêtres puissent être conduits à soigner des femmes, mais Frank les rejette, arguant du fait que les prêtres sont mariés dans l'Église orthodoxe et que leurs épouses pourraient servir d'auxiliaires en tant que sages-femmes. ${ }^{33}$

30 Voir ci-dessous, document II, p. 260.

31 Debono 1997, 143, 224.

32 Heller 1976,366, n. 34. Il s'agit des conciles de Clermont (1130), Reims (1133), Latran (1139), Tours (1163), Montpellier (1195).

33 Heller 1976, 377. 


\section{Le prêtre-médecin dans la formation médicale russe}

L'ensemble du projet de Frank a fait l'objet d'une étude détaillée dans l'article de Robert Heller qui reprend le concept des prêtres-médecins dans l'ensemble de l'Europe au siècle des Lumières; l'auteur y rapporte à la fois la lettre du comte Potocki, ainsi que la transcription de l'oukase impérial. En revanche, il ne mentionne pas la réponse que Frank a faite au comte; voici ce qu'il déclare:

Il n'existe aucune preuve que Frank ait jamais élaboré le programme d'études demandé par le comte Potocki. Il n'y a jamais fait allusion. Il ne fait aucun doute que ce projet avait suivi le même chemin que de nombreux autres plans ambitieux parrainés par Alexandre I ${ }^{\text {er }}$, qui étaient soit impossibles à réaliser compte tenu de l'état rétrograde du pays ou étouffés par la résistance de la noblesse, de l'Église ou de la bureaucratie. ${ }^{34}$

En réalité, et c'est là l'originalité du document III rapporté ci-dessous, Frank a répondu au comte et fait des propositions pour la mise en place d'un cursus pour ces fameux prêtres-médecins. La missive est une version antérieure à ce qu'il a par la suite publié dans son ouvrage System einer vollständigen medicinischen Polizey. ${ }^{35}$ Cependant des différences apparaissent nettement. Dans la version publiée du projet, le cursus des étudiants doit comporter trois années, mais dans la version manuscrite, il n'en mentionne que deux, même s'il laisse la possibilité aux autorités de prolonger éventuellement les études:

J'avoue, que le temps de deux années est trop court pour former des médecins sur lesquels l'on puisse compter beaucoup dans l'exercice de l'art. Si les jeunes Ecclésiastiques passoient trois ou quatre années dans les seminaires: alors il seroit bien facile de distribuer les matières de manière à etre enseignées sans tant de précipitation; et dans ce cas il ne faudroit pas employer moins de deux années pour instruire ces élêves dans les écoles cliniques de médecine et de chirurgie. ${ }^{36}$

Le plan des études, de ce fait, est différent dans les deux versions puisqu'il faut ajuster et harmoniser sans pour autant que dans la proposition initiale de Frank la formation soit amputée. Voici le tableau que l'on peut dresser à partir des deux versions:

34 Heller 1976, 376.

35 Frank 1817, vol. VI/2, 451-455.

36 Voir ci-dessous, document III, p. 261. 


\begin{tabular}{|c|c|}
\hline $\begin{array}{l}\text { System einer vollständigen } \\
\text { medicinischen Polizey }\end{array}$ & Lettre de Frank n III \\
\hline $1_{\text {ère année du cursus }}$ & $\begin{array}{l}1^{\text {ère }} \text { année du cursus } \\
\text { Anatomie }=3 \text { mois (hiver) + bases de la physiologie }\end{array}$ \\
\hline Physiologie & $\begin{array}{l}\text { Répétition physiologie }+ \text { pathologie }+ \text { hygiène } \\
+1 \text { heure quotidienne botanique }=9 \text { mois }\end{array}$ \\
\hline Pathologie & Total $=12$ mois \\
\hline $2^{\text {ème }}$ année du cursus & $2^{\text {ime }}$ année du cursus \\
\hline Principes de la thérapeutique générale & $\begin{array}{l}\text { Thérapeutique générale + Matière médicale } \\
\text { (enseignement pratique en boutique) } \\
+ \text { connaissance des instruments }=5 \text { mois }\end{array}$ \\
\hline Matière médicale & $\begin{array}{l}\text { Hôpital enseignement pratique au chevet } \\
\text { du malade }+ \text { Techniques de réanimation }=5 \text { mois }\end{array}$ \\
\hline $\begin{array}{l}\text { Connaissance des instruments } \\
\text { Faire des bandages }\end{array}$ & Total $=10$ mois \\
\hline
\end{tabular}

$3^{\text {ème }}$ année du cursus

Thérapeutique pratique

Chevet du malade

Techniques de réanimation

On le voit, le projet initial a considérablement concentré au cours de la seconde année du cursus ce qui est développé dans le cadre d'une troisième année dans l'ouvrage. Il s'agissait vraisemblablement de ne pas trop effrayer les autorités avec un cursus trop long qui venait s'ajouter à celui que les candidats avaient initialement accompli en théologie. Néanmoins, Frank a conscience, dans la version définitive de son projet, que la part qui doit être consacrée à l'enseignement pratique au chevet du malade est insuffisante et c'est la raison pour laquelle il prend la peine d'ajouter une année supplémentaire. Il semble que la proposition de Frank pour les prêtres-médecins s'inspire du statut d'officier de santé en France et dont il a pu avoir connaissance. ${ }^{37}$

37 C'est sous le Consulat avec la loi du 19 ventôse an XI (10 mars 1803) que sont instaurées des écoles de médecine. On abolit la distinction entre médecins et chirurgiens, la médecine comporte alors deux niveaux: celui des docteurs, issus des écoles, devenues facultés en 1808, et dont le titre confère le droit d'exercer la médecine et la chirurgie sur tout le territoire, et celui des officiers de santé, pratiquant une médecine à l'échelle seule du département et qui ont suivi des études plus brèves, à l'instar de Charles Bovary, dans le roman de Gustave Flaubert, Madame Bovary. Les officiers de santé sont reçus par des jurys médicaux dans les départements. Les officiers de santé ne jouissaient pas de tous les privilèges des médecins. Leur exercice était limité au département dans lequel ils étaient inscrits. Ils n'avaient pas le droit d'effectuer certains actes et ne pouvaient pas avoir accès aux fonctions de médecin hospitalier ou d'expert. Ce deuxième ordre était réservé à des praticiens, non titulaires du baccalauréat, ayant suivi des stages dans des hôpitaux ou chez des praticiens et ayant fait preuve de leurs connaissances, lors d'un examen soutenu devant un jury départemental (Ackerknecht 1986, Corlieu 1896 - dont l'ouvrage très complet détaille notamment l'organisation de la scolarité et le contenu des examens -, Coury 1968, Huguet 1991). 
Le doctorat en médecine ou chirurgie était obtenu, en France, après quatre années d'études, terminées par cinq examens et une thèse en français ou en latin. Pour devenir officier de santé, il fallait passer trois années dans une école de médecine, ou bien cinq dans un hôpital civil ou militaire, ou bien six auprès d'un docteur, sanctionnées par un examen devant un jury médical départemental. L'officier de santé ne pouvait exercer que dans les limites du département où il avait été reçu.

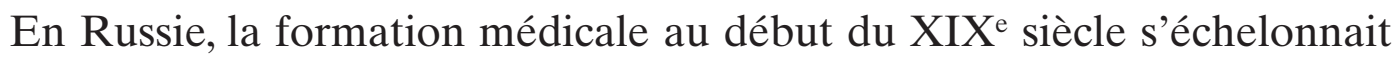
sur une période de cinq années; on y enseignait six matières: ${ }^{38}$

1. Anatomie, physiologie et jurisprudence médicale,

2. Pathologie, thérapie et pratique,

3. Pharmacologie,

4. Chirurgie,

5. Obstétrique,

6. Pratique vétérinaire.

On le voit, la formation russe était plus longue que celle qui se pratiquait en France à la même période.

Il ne semble pas que les propositions de Frank aient eu un commencement de réalisation - il se plaint au contraire de se voir enlever par l'armée ses étudiants à peine formés. Supportant mal les rigueurs du climat, il reviendra à Vienne en juillet 1808. Il conservera toutefois le souvenir de cet épisode pétersbourgeois et en rendra compte dans son System einer vollständigen medicinischen Polizey.

Les documents suivants sont conservés par les Archives historiques de l'État de Lituanie (LVIA) à Vilnius, sous la cote F. 1511, op. I/53, ff. 253-264, dans le fonds des professeurs de l'Université de Vilna (1738-1858). Le document III est autographe; c'est l'état préparatoire de la version mise au net qui fut sans doute adressée à Séverin Potocki. Elle n'a pas été retrouvée. Les nombreuses corrections portées aux lignes qui décrivent le «plan d'enseignement» montrent l'importance que Frank lui donnait. Conformément à l'usage aujourd'hui répandu dans l'édition scientifique, les textes sont établis en respectant la graphie originelle.

38 Debono 1997, 89. 


\section{Document I: La lettre de Séverin Potocki à Jean-Pierre Frank}

\section{Monsieur!}

Sa Majesté L'Empereur ayant désiré procurer des moyens de secours aux habitans des campagnes dans leurs maladies, a ordonné par Oukase que les jeunes éclésiastiques fussent instruits dans les principes de la médecine; ce qui a aussi été éxécuté autant que possible dans les divers séminaires. Cepandant ces établissements ayant en général des fonds bornés, Monseigneur L'Évêque de Harkoff ${ }^{39}$ a permis aux étudiants du sien, de suivre les cours de l'Univérsité, ${ }^{40}$ naturellement bien plus complets; mais de ce côté il se présente encor plus d'une difficulté: 1 qu'en suivant toutes les branches de la science, ces jeunes gens seroient necéssairement détournés du but principal de leurs études; 2 qu'en n'en suivant que quelques-unes, on courroit risque d'en faire des demi-médecins, qui feroient plus du mal que de bien, $\&$. Il semble donc qu'il seroit éssentiel de tracer tant pour les connoissances préparatoires, telle que la Physique, la botanique et la chymie que pour celles de la médecine en elle même, un plan d'enseignement, qui leur apprit juste ce qu'il faut et rien audela, qui les mit à même de combattre les préjugés du peuple et de porter remede dans des cas ordinaires, qui leur montrat surtout les bornes, dans les quelles ils doivent se renfermer pour ne jamais devenir dangereux. Hors [sic] ce plan d'enseignement qui peut le tracer mieux que Vous, Monsieur, qui vous étes fait également un nom et par vos ouvrages, si connus ${ }^{41}$ et votre pratique si étendue; j'espere donc que Vous voudrez bien me pardonner mon importunité et ne réfuserez pas de Vous charger de ce travail si facile pour Vous et qui n'en sera pas moins utile à la Patrie, que Vous venez d'adopter. - Je Vous envoye ci joint une traduction de l'Oukase, qui Vous instruira en detail des vües de Notre bienfaisant Souverain; j'attendrai avec impatience Votre reponse et Vous prier [sic] d'agreer l'assurance de la consideration la plus distingué[e], avec laquelle j'ai l'honneur d'étre Monsieur

Votre tres humble et tres Obeissant

$$
\begin{gathered}
\text { Serviteur } \\
\mathrm{C}^{\text {te }} \text { Severin Potocki }{ }^{42}
\end{gathered}
$$

39 Kharkov (auj. Kharkiv) en Ukraine orientale.

40 L'Université de Kharkov fut fondée en 1804 et ouvrit le 29 janvier 1805.

41 On peut citer System einer vollständigen medicinischen Polizey [...] (Mannheim 1780-1790), Plan d'école clinique ou méthode d'enseigner la pratique de la médecine dans un hôpital académique [...] (Vienne 1790), Traité sur la manière d'élever sainement les enfants (Paris 1798).

42 Les trois dernières lignes sont autographes. 


\section{Document II: L'oukase d'Alexandre I ${ }^{\mathrm{er}}$}

Ayant le désir de rémplacer dans les villages le défaut d'établissemens qui régardent la santé du peuple et de procurer à leurs habitans les moyens du soulagement nécessaire dans les maux corporels par le secours de leurs prêtres, je joins ici la copie de l'Ukase, donné au Saint Synode, concernant l'introduction des connoissances nécéssaires pour cet objet dans les séminaires de l'Empire, en préscrivant au Sénat Dirigant ${ }^{43}$ de donner au Collège de Médecine toutes les instructions possibles et de contribuer à l'avenir de toutes ces forçes dans tous les cas, qui éxigéront son secours.

Il est connû qu'il n'y a point des médecins dans les villages, et que les accidens les moins graves y déviennent mortels par l'ignorance, par des prejugés invétérés et par la manière de traiter les maladies, toute-à-fait contraire tant à leur nature qu'au bon sens. En désirant de donner dans cette vüe aux habitans de la campagne le secours aussi simple que conforme à leur manière de vivre, je pense que le moyen le plus convenable d'atteindre ce but, serait de confier ces soins aux prêtres des villages en leur procurant toute la facilité à pouvoir réunir avec les connoissances conformes à leur vocation celles de la médecine. Pour mêttre en éxécution ce plan, il est nécéssaire que le Saint Synode, àfin de contribuer à cet établissement de charité, fasse les dispositions suivantes: 1 de joindre aux mêmes fraix un certain nombre d'élèves au cinquant étudians, ${ }^{44}$ que les Séminaires énvoyent dans les écoles de médecine, àfin que ces élèves ayant finis leurs cours d'étude et de rétour dans leurs Diocèses, remplissent les places des précepteurs de ces sciences dans les séminaires. 2 Dans l'intervalle du tems dans lequel ces maitres seront formés, le College de médecine doit composer une instruction abregée mais claire de la manière de traiter les maladies les plus ordinaires aux habitans de la campagne, en y joing[n]ant la déscription des rémèdes, qui peuvent être préparés dans les villages mêmes; après en avoir imprimé la quantité nécéssaire d'éxemplaires le Collège doit les énvoyer au Saint Synode, qui les faira distribuer dans les Séminaires et dans toutes les églisses parroissiales pour

43 «Le Sénat dirigeant de Russie peut être regardé comme le premier corps de cet empire [...] Ce sénat veille à l'exécution des lois, surveille la rentrée et l'emploi des deniers publics, promulgue les lois et les édits rendus par l'empereur, nomme à la plupart des grands emplois et juge en dernier ressort toutes les causes et tous les conflits.» (Migne 1859, 809).

44 «Quoique les cinquante fils de prêtres grecs, que le Saint Synode fournissait tous les ans à l'Académie médico-chirurgicale, eussent reçu quelque instruction gymnasiale, il s'en fallait cependant beaucoup qu'ils fussent convenablement préparés à passer directement à l'étude des sciences physico-médicales. [...] Vu l'impossibilité d'enseigner en si peu de temps les sciences médicales, mon père proposa d'y consacrer cinq ans. Cette proposition fut aussi rejetée à cause du besoin urgent qu'on avait d'officiers de santé pour les armées.» (Mémoires biographiques, ch. L). 
l'usage convenable. 3 Outre cela le Collège préscrira aux Comités de médecine /:Управам:/ de chaque Gouvérnement, ${ }^{45}$ d'énvoyer à la démande des Archevêques de Diocèses, quelques uns de ses mémbres les plus experts ou des médecins qui se trouvent dans les autres villes de Gouvérnement pour énseigner les élémens de médecine dans les séminaires juscequ'à ce qu'ils seront fournis de leurs propres maitres, et de leur prêter à l'avenir tous les secours, qui en dépendent pour éténdre ces connoissances parmi le Clergé. 4 Conformément à ces dispositions principalles, j'ai ordonné au Sénat Dirigant de donner les instructions convenables à tous ceux que cette affaire régarde, et pour préndre les mésures de détails qui à l'avenir pourroient être nécessaires à la meilleure organisation de cette partie, $\mathrm{j}$ 'ai confié cette affaire aux soins particulièrs d'Ambroise ${ }^{46}$ Métropolite de Nowgorod et $S^{t}$ Pétérsbourg, en lui préscrivant de communiquer immédiatement à ce sujet avec le Comte de Wasilieff, ${ }^{47}$ le Gouverneur Général de Collège de médecine. 5 Pour ce qui regarde les moyens d'éncourager les prêtres à faire prospérer cet établissement utile et l'assignation de la somme nécéssaire pour l'éntrétien des maîtres et l'achât des instrumens, le Saint Synode après avoir fait les rénseignemens nécéssaires, me les préséntera dans son tems.

\title{
Document III: Les propositions faites au Comte Potocki
}

\author{
à S. E. Mr. le Comte Severin Potocki, Senateur à Petersbourg \\ et Curateur de l'Université de Charkoff.
}

Monsieur le Comte!

Votre Excellence m'a fait l'honeur de réchercher mon opinion sur la meilleure manière d'exécuter un Oukase paternel de Sa Majesté Impériale, qui ordonne, que les jeunes Ecclésiastiques soient instruits dans les principes de la médecine, pour suppléer ainsi au défaut d'établissements rélatifs à la conservation de la santé du peuple distribué dans les vastes domaines de la Russie.

Vous savés, Monsieur le Comte, ${ }^{48}$ que sans la connaissance de cet Oukase, j'ai fait voir dans un[e] harangue académique, prononcée en 1803 par-devant

45 Les Comités de médecine (медицинские управы) existaient dans tous les gouvernements de l'empire de Russie depuis 1797. Ces comités étaient chargés de la lutte contre les infections, du contrôle des pharmacies et des hôpitaux, de l'expertise médico-légale (Information aimablement fournie par M. Sergueï Karp).

46 Ambroise Podobedov (1742-1818), archevêque en 1799, métropolite en 1801.

47 Alexeï Ivan. Vassiliev (1742-1807), administrateur du Collège de médecine, ministre des Finances.

48 Biffé (Literaturhinweis?): «que sans la publication». 
l'Université de Vienne en Autriche, que la médecine, dans tous les tems et chés tous les grands ${ }^{49}$ Peuples de l'Antiquité, a toujours été cultivée avec soin et exercée avec zèle du Clergé; que les prêtres chrétiens dans les prémiers siècles ont toujours suivi à la lettre le Commandement de leur fondateur divin qui leur enjoignoit de guérir les malades ${ }^{50}$ et que ce n'est que la politique des chefs de l'Église latine et de quelques uns des Conçils, qui, au commencement du douzième siècle /A. 1131-1139/, ont exclu les prêtres de tout exercice, prémierement de la chirurgie, et enfin même de celui de la médecine. Cette prohibition étoit bien une suite nécessaire de celle, que la même Église avoit portée quelques siècles auparavant quant au mariage des prêtres: lesquels, forcés enfin, sans exception, au célibat, n'étoient probablement plus aussi irréprochables dans leur conduite vis-à-vis le sexe malade confié à leurs soins, que ne l'avoient été les prêtres mariés dans les tems antérieurs. Lorsque de mon coté je démontrois la nécessité de recourir de nouveau à l'ancien usage d'employer les curés de la campagne pour suppléer au défaut des médecins: ${ }^{51}$ un Évêque zélé de la france publia une pastorale digne des prémiers siècles chrétiens, pour faire envisager à son clergé subordonné le besoin $^{52}$ de se vouer en même tems à la médecine pour secourir les habitants de la campagne privés de secours et abbandonnés cruellement à leur sort dans les maladies les plus dangereuses. ${ }^{53}$

Quelle devoit être ma satisfaction, Monsieur le Comte, en apprennant, que le grand Souverain de la Russie, par un mouvement spontané de son cœur bienfaisant ${ }^{54}$ avoit daigné mettre en exécution, ce que, comme membre de l'Église catholique, je n'avois osé conseiller qu'avec le plus grand risque de déplaire à la Cour de Rome comme il m'étoit déjà arrivé vingt années auparavant par mon ouvrage de Police médicale, ouvrage dans lequel j'avois enseigné, que l'Église latine, en suivant l'exemple des prémiers siecles chrétiens, et celui de l'Église grècque, toujours fidèle à ce modèle, seroit applaudie de tous les Êtres pensants, si elle consentit à rétablir le clergé séculier dans $\operatorname{ces}^{55}$ droits naturels au mariage!

49 Ajouté en marge.

50 Matthieu, 10, 8.

51 «Lorsque de mon coté $[\ldots]$ » ajouté en marge remplace «C'étoit à peu près dans le même tems qu'».

52 «le besoin» remplace «la nécessité».

53 Il s'agit probablement de la lettre pastorale sur la vaccine envoyée aux curés de son diocèse par l'archevêque de Besançon, Claude Le Coz (1740-1815), dans laquelle, après avoir rappelé l'exemple de Jésus, de saint Pierre «dont l'ombre seule guérissoit les infirmes», de saint Luc «qui étoit médecin», il leur montre «que jusqu'à l'an 1452, tous les professeurs en médecine de l'université de Paris firent profession de la vie cléricale et de la continence» (Annales 1804, t. 1, 428-429).

54 «bienfaisant» ajouté en marge remplace quelques mots que je n'ai pu lire.

55 Surcharge «ses». 
Je sens bien, comme V. E. le pensera, les difficultées de rendre les prêtres à une science qu'ils n'auroient jamais dû abandonner, et laquelle, de son côté, n'avoit qu'à perdre par leur séparation mal entendue. Ces difficultées doivent être plus grandes en Russie, que partout ailleurs, où les occasions de s'approprier les principes de la médecine sont de beaucoup plus nombreuses que dans cet Empire. Mais quand un Souverain aussi éclairé que puissant veut le bien que la réligion et la raison approuvent, quelques puissent être les obstacles qui s'y opposent, ils disparoissent comme les brouillards sont dissipés par l'action du soleil. Déjà notre Clergé séculier fournit à la médecine une partie de ses Enfans, sans les quels la Russie seroit encore plus privée qu'elle ne l'est de médecins. ${ }^{56}$ Encore un pas, et ce grand Empire pourra se vanter non seulement d'avoir rempli cette lacune; mais aussi d'avoir donné l'exemple à l'Europe dans l'administration de la médecine quant au peuple de la campagne la plus naturelle.

Une seule considération m'effraye, Monsieur le Comte, et Vous même Vous l'avés faite, cette considération: c'est que, si par un nouvel ordre des choses, on ne procuroit à la Russie dans ces prêtres que des médecins à demi leur assistance seroit peutêtre plus dangereuse aux malades, que ne l'est le défaut même des personnes de l'art. Malheureusement il s'en faut de beaucoup, que tous les médecins du gouvernement soient assés au fait de leur science, pour en enseigner les meilleurs principes aux autres; et ceux même qui ont fait des études régulières en médecine, ne pouvant leur donner pour l'ordinair[e] que quatre années qu'ils passent à l'académie médico-chirurgicale, ou aux Universités, sont encore trop novices dans leur art, pour leur confier la charge d'enseigner une doctrine qui exige une si longue expérience. Je n'ignore pas qu'on se dit: «qu'il suffit aux prêtres, qu'on leur enseigne ce qu'il leur faut pour être en état de combattre les préjugés du peuple, et pour porter remède à celuiçi dans les cas ordinaires en se contenant dans les bornes qui leur seront prescrites dans leur instruction;»- mais, accordé que les prêtres, instruits dans les prémiers principes de la médecine, pourront déraçinner un très grand nombre de préjugés nuisibles à la santé et à la conservation du peuple, - même les cas ordinaires des maladies exigent souvent une dextérité peu commune pour leur traitement; et comment l'homme isolé, pourvu de quelques connoissances en médecine, pourra-t-il se réfuser

56 «L'académie médico-chirurgicale, qui devait d'autant plus m'intéresser que mon père y remplissait les doubles fonctions de recteur et de professeur. Cette académie, ou plutôt cette école générale des sciences médicales, se trouvait placée dans un superbe édifice. Elle comptait deux cents élèves, pour la plupart fils de prêtres grecs, le clergé russe étant obligé de fournir par année un contingent de cent cinquante jeunes gens à l'établissement en question, pour y être élevés et instruits aux frais de la Couronne, pendant quatre ans, à condition de servir ensuite six ou sept ans dans les armées de terre ou de mer, et dans le civil.» (Mémoires biographiques, ch. XLIX). 
aux malheureux, qui dans les maladies les plus difficiles ne trouvent pas même à 300 Werstes un médecin plus instruit? Aussi les baigneurs et barbiers de l'Allemagne ${ }^{57}$ ont leur instruction, qui leur défend l'exercice de la médecine dans les maladies de conséquence, mais savent ils toujours distinguer le degrés de danger où se trouvent leurs malades?... et quand même ils le connoîtroient, les hommes ne sont ils pas assés généralement ou aveuglés par l'amour propre, ou séduits par l'appareil du gain ou de la renommée qu'ils attendent de leurs transgressions?

Vous attendés sans doute, Monsieur le Comte, que je vous propose le moyen de prévenir ces conséquences dangereuses, et j'en aurois un de tout prèt, si l'État crut pouvoir

1 permettre à tout le Clergé séculier demeurant à la campagne, d'exercer librement la médecine dans laquelle il auroit été approuvé d'après les lois de l'Empire.

2 accorder aux médecins, lorsqu'ils le desireroient, des places de prêtres de campagne, quand ils auroient satisfait aux règles prescrites pour les études sacrées.

De cette manière le nombre du Clergé ne souffriroit plus de subtraction ${ }^{58}$ par l'envoi de ses fils à l'étude de la médecine: subtraction, qui doit lui être d'autant plus sensible, qu'il s'en faut de beaucoup qu'il y ait en Russie assés de prêtres pour l'instruction réligieuse et la conduite morale du peuple; et en même tems l'État ne manqueroit plus, comme il manque jusqu'içi, et comme il manquera encore bien longtems, de sujets bien instruits pour l'exercice de la médecine.

Mais, supposé, que pour se réfuser ce remède héroique/: le seul propre à la grandeur du mal que souffrent les differents gouvernements de la Russie par le défaut de médecins:/ l'on n'eût d'autres raisons, que la crainte, que les études sacrées ne souffrissent par le tems employé à celles de la médecine;cette crainte est elle bien assés fondée et ${ }^{59}$ justifiée par l'expérience et par la raison même?... Les prémiers prêtres chrétiens, - le diacre et martyr, saint Papilus, sous l'Empereur Décien, - le prêtre et martyr, $\mathrm{S}^{\mathrm{t}}$ Zenobien sous l'Empereur Dioclétien ${ }^{60}$ - Dyonise le Lévite, au commençement du cinquième

57 «La Chirurgie ainsi dégradée étoit tombée exclusivement entre des mains grossières, qui ne pouvoient ni l'exercer, ni la perfectionner. Ici [à Vienne], \& presque dans toute l'Allemagne, abandonnée à des barbiers, à des baigneurs ignares, dénués de théorie, incapables de s'éclairer même par la pratique, charlatans dangereux, plutôt que Chirurgiens, ce vaste empire en étoit le tombeau, plutôt que le théatre.» (Brambilla 1787, 36-37).

58 Le mot est rare, mais apparaît encore au XVIII ${ }^{\mathrm{e}}$ siècle.

59 En interligne: «fondée et».

60 «Saint Papilus, d'abord illustre médecin, puis diacre et martyr à Pergame en Asie, l'an 164, sous la persécution de Marc-Antonin et de Commode. / Saint Zenobius, d'Egée en Cilicie, médecin, évêque, martyr sous Dioclétien.» (Montrond 1852, 37). 
siècle, - le Diacre Elpidius Rusticus, ${ }^{61}$ Eusebius,${ }^{62}$ Zeno, Biasius et Théodo${ }^{t u s}{ }^{63}$ pour avoir été médecins et prêtres en meme tems, étoient ils moins instruits, moins zélés dans la foie, que ne le sont la pluspart de nos prêtres? ou leur réputation médicale ne leur a-t-elle pas plâné [sic] le chemin pour prêcher avec plus d'onction une réligion charitable et bienfaisante? L'étude de la nature et de son chef-d'œuvre, l'homme, n'est-elle pas faite pour exciter, en qui la cultive, l'admiration et une vénération plus sentie du Créateur? La perscrutation ${ }^{64}$ des vertues, que la providence a prodiguées aux plantes et aux minéraux pour la conservation de notre espèce, seroit elle bien faite pour affoiblir les connoîssances d'une réligion fondée sur l'exercice de la charité?... Jettons les yeux sur le grand nombre d'Écclésiastiques de différentes réligions, qui, outre la réputation de prêtres éminemment instruits et vertueux, se sont distingués encore autant par leurs vastes connoîssances dans les sciences profanes, dans les mathématiques, dans la physique, dans l'histoire naturelle, dans les belles-lettres, et même dans l'agriculture, - et confessons, que ${ }^{65} 1$ 'assertion d'une incompâtibilité des études sacrées avec celles de la médecine, est destituée de fondement. Dans toutes les réligions, le désœuvrement des prêtres de la campagne, les dispose à des vices, qui ne prennent guer[e] raçine dans l'homme assés occuppé des sciences. Les prêtres protestants sont en général meilleurs citoyens, plus instruits, et plus morigénés, ${ }^{66}$ que la pluspart des prêtres d'une Église qui a cru devoir porter l'anathème sur ceux de ses membres, qui auroient assés de connoîssances et assés de bonne volonté, pour sauver la vie à leurs ouailles; ou qui oseroient donner des enfans légitimes et bien éduqués à leur patrie.

L'on conviendra peutêtre avec moi, que le prêtre déjà formé dans les séminair[e]s pourra bien partager un jour son tems entre les études sacrées et les sciences profanes; mais l'on voudra toujours soutenir: «que les jeunes Écclésiastiques, en suivant toutes les branches de la science médicale, seroient nécessairement détournés du but principal de leurs études.» - Peutêtre qu'un autre répondroit à cette objection que ne voulant consulter que l'expérience, il paroîtroit que ce que la pluspart des prêtres de la campagne ont rapporté jusqu'içi en connoîssances des séminair[e]s, se laisseroit fort bien combiner avec l'Étude la plus sérieuse de la médecine, quant à moi, je conviendrai, que sans doute l'on ne sauroit prétendre que les séminaristes se vouent à toutes

61 Voir sur le diacre Denis (Levita Dionysius), Ceillier 1748, t. XV,424; sur Elpidius, ibid.,t. XVI, 181.

62 «Saint Eusèbe, médecin grec, pape, martyr sous l'empereur Maxence.» (Montrond 1852, 37).

63 Sur les trois derniers, voir Rohlfs 1880, vol. 2,156.

64 Recherche approfondie.

65 «et confessons, que» ajouté en marge.

$66 \mathrm{Au}$ sens de formés aux bonnes mœurs (Trévoux). 
les branches de la médecine dans la même extension, que les jeunes gens qui ont choisi cette science pour leur unique occuppation, et c'est là précisément l'objet de la question, savoir:

«Quel doit être le mode d'instruction des jeunes Écclésiastiques dans la science médicale, pour que, sans être détournés par celle çi du but principal de leurs études, ils y acquierrent assés de connoîssances pour traiter, au défaut de médecins plus formés, les maladies ordinaires du peuple de la campagne avec succès?»

Il paroit bien contradictoir[e] de joindre l'étude de la médecine comme accessoire à celle de quelque science que ce soit qui, comme elle, n'embrasse pas toute la nature; cependant, avouons, qu'en séparant de la science médicale, la connoîssance des faits, de celle des opinions: la masse de ce qui est essentiel en est prodigieusement dimminuée.

L'étude de la médecine, comme celle de toute autre science, présuppose des connoîssances suffisantes dans la philosophie, dans la physique, et dans l'histoire naturelle.

Les parties principales de la science médicale elle même, sont

1. l'anatomie,

2. la physiologie,

3. la pathologie,

4. la matière médicale et l'hygiene

5. la thérapie médicale et chirurgicale.

L'Anatomie en général est une partie de l'histoire naturelle; celle de l'homme, doit occupper de préférence le médecin. Il s'en faut de beaucoup que le[s] progrès immenses que nous avons faits en cette partie, ayent enrichi en proportion l'art de guérir. L'on ne sauroit donc vouloir enseigner l'anatomie dans tous ses détails à ceux qui doivent se contenter du necessair [e].

Les parties de l'anatomie, sont: a) l'ostéologie; b) la myologie;c) la splanchnologie; d) l'angiologie; e) la nevrologie; f) l'adénologie; et g) la syndesmologie.

L'ostéologie, ou la connoîssance des os, fait la base de l'anatomie; cependant le médecin praticien peut se contenter d'une idée nette de la structure, de la forme et de la connexion de ces parties sans y entrer dans des détailles trop minutieux.

La myologie, ou la connoîssance des muscles, interesse infiniment le praticien, surtout le chirurgien, pour ce qui regarde la situation, l'usage ${ }^{67}$ de ces instruments du mouvement animal, et la direction de leurs fibres, ainsi que de celle des vaisseaux et des nerfs qui parcourent leurs substances. Quant aux 67 En interligne. 
insertions de tous les tendons, leur connoissance est aussi peu essentielle, que celle de leurs points d'attachement dans l'ostéologie.

Une partie de l'anatomie bien essentielle c'est la splanchnologie, ou la doctrine des viscères. Elle doit être donnée aux élêves avec tous les détails dont elle est susceptible. Quant aux cerveau néanmoins, il n'y a jusqu'içi que la considération de ses parties principales, de ses enveloppes, et de l'origine des nerfs qu'il fournit, qui mérite l'attention du praticien.

La doctrine des vaisseaux, tant sanguins que lymphatiques /angiologie/ est de toute nécessité. L'on ne sauroit donner une bonne splanchnologie sans des détails exactes sur ce systême; et surtout les artères et veines dont la lésion peut devenir dangereuse, doivent être parfaitement connus au chirurgien. ${ }^{68}$ Quant à leurs ${ }^{69}$ ramifications plus fines, elles ne méritent pas que les élêves, dont il s'agit, s'en occuppent beaucoup.

La nevrologie, ou doctrine des nerfs, exige la plus grande attention du praticien, et sa dignité ne permet guer[e] de la donner trop superficiellement.

La science des glandes, ou l'adenologie, fait une partie de la splanchnologie très essentielle, et même les glandes qui ne concernent pas celleçi, méritent d'être démontrées.

La syndesmologie, ou la doctrine des ligaments est plus nécessaire aux chirurgiens qu'aux médecins. Les capsules cependant et les ligaments, ${ }^{70}$ qui enveloppent les principales articulations, leur doivent être clairement exposés.

C'est dans les leçons anatomiques, ${ }^{71}$ données complètement dans le cours d'un hiver, que doit être jetté la base de la physiologie. À la fin du cours anatomique, ou du printems jusqu'en automne, les élêves peuvent répéter brièvement cette dernière, en la combinant chaque fois avec des réflexions pathologiques. C'est dans la physiologie, qu'il convient d'exposer les règles diététiques ${ }^{72}$ qui concernent l'homme bien portant, ou l'hygiène..$^{73}$ Une heure de l'après midi doit être employée pour la connoissance ${ }^{74}$ des principales $^{75}$ plantes médicales, en y ajoutant toujours leur manière d'agir sur le corps de l'homme, et la mannière de les employer. ${ }^{76}$ Les végéteaux venimeux ${ }^{77}$ qui

68 Remplace «médecin».

69 «à leurs» remplace «aux».

70 «cependant et les ligaments» remplace «et ligaments cependant».

71 Biffé: «qui doivent être».

72 Biffé: «et de celles de l'hygiene qui».

73 «C'est dans la physiologie [...]» ajouté en marge.

74 «systématique» ajouté en marge, puis biffé.

75 Ajouté en marge.

76 «et la mannière de les employer.» ajouté en marge.

77 «Les végéteaux venimeux» remplace «Les plantes venimeuses dont». 
croissent dans le païs, et y occasionnent souvent des effets pernicieux aux hommes et aux animaux domestiques, ne doivent pas être oubliés dans les ostensions. ${ }^{78}$ De cette manière les élêves auront appris dans la prémière année les éléments de l'anatomie, de la physiologie, de la pathologie, et de la botanique médicale.

Dans la seconde année, pendant les cinque ${ }^{79}$ prémiers mois, l'on expliquera aux élêves les règles de la thérapie générale, aux quelles l'on ajoutera ${ }^{80}$ la connoîssance de la diéte des malades, celle ${ }^{81}$ des principaux médicaments, ${ }^{82}$ de leurs doses et de la manière d'en préparer les plus simples et de les ${ }^{83}$ expédier. Cette dernière doctrine se donnera le mieux dans une bonne ${ }^{84}$ pharmacie du lieu. Une heure de l'après midi ${ }^{85}$ doit être employée pour enseigner aux élêves la manière de faire une saignée, de mettre des lavements, d'appliquer des $^{86}$ ventouses, des sangsues, des sinapismes, des vésicatoirs; d'arrêter le sang dans les hémorrhagies dangereuses; de remettre les hernies, les descentes, ${ }^{87}$ les luxations, les fractures, d'ouvrir un abscès. Les cinques mois qui suivent, les élêves seront conduits dans un hôpital, où leurs seront montrées et expliquées les principales maladies populaires, avec la manière la plus simple de les traiter. Outre cette leçon, on leur donnera chaque après midi une autre sur le traitement des asphyxies, des maladies causées par un empoisonnement accidentel. Surtout on enseignera aux élêves de bien questionner les malades pour pouvoir en écrire les rapports sur des maladies plus graves, ${ }^{88}$ qu'ils devront communiquer aux medecins les plus voisins. L'on tâchera ${ }^{89}$ principalement de leur faire entrevoir les limites lesquelles ${ }^{90}$ ils ne sauroient passer sans aggraver leur conscience, et sans blesser les loix.

Ce cours d'études comprend à la vérité toutes les parties les plus essentielles de l'art de la médecine; mais comme il s'agit plus d'imprimer profondément ${ }^{91}$ à la mémoire ces objets, que d'en donner simplement un apperçu: j'avoue, que le tems de deux années est trop court pour former des médecins ${ }^{92}$

78 Même si le sens est clair, le mot appartient au vocabulaire religieux.

79 Remplace «quatre».

80 Biffé: «les».

81 «de la diéte des malades, celle» ajouté en marge.

82 Biffé: «celles».

83 «d'en préparer les plus simples et de les» remplace «de les prescrire ou de les».

84 En interligne.

85 Remplace peut-être «diner».

86 «'appliquer des» remplace «des».

87 En interligne: «les descentes,».

88 «sur des maladies plus graves,» ajouté en marge.

89 Remplace «insistera».

90 Remplace «au dela desquels».

91 En interligne.

92 Biffé: «tant soit». 
sur lesquels l'on puisse compter beaucoup ${ }^{93}$ dans l'exercice de l'art. Si les jeunes Ecclésiastiques passoient trois ou quatre années dans les seminaires: alors il seroit bien facile de distribuer les matières de manière à etre enseignées sans tant de précipitation; et dans ce cas il ne faudroit pas employer moins de deux années pour instruire ces élêves dans les écoles cliniques de médecine et de chirurgie.

Comme les jeunes gens ${ }^{94}$ qui se vouent entièrement à la médecine ne sauroient être instruits aussi rapidement ${ }^{95}$ que ceux, qui ne sont destinés que pour $^{96}$ être un jour des médecins supplémentaires: il ${ }^{17}$ seroit fort à desirer, qu'un Seul Professeur put donner toutes les leçons de médecine ${ }^{98}$ dont j'ai parlé aux jeunes Ecclésiastiques. Le même homme enseignant toutes les parties d'une science aussi compliquée que ${ }^{99}$ la médecine, trouvera le moyen de mieux les enchainer, ${ }^{100}$ d'éviter ${ }^{101}$ toutes les répétitions superflues, ${ }^{102}$ toute contradiction, et de déduir[e] naturellement chaqu'assertion ${ }^{103}$ nouvelle des principes renfermés ${ }^{104}$ dans les leçons précédentes. En outre les Élèves s'habitueront plus facilement à comprendre ses explications; et sa manière de s'énoncer sera plus à la portée de ceux qui l'ont fréquenté pendant la prémière année. Mais il faudroit que cet homme ne fut pas lui même peu versé dans la médecine, et qu'il fut doué d'assés d'expérience, ${ }^{105}$ pour pouvoir ${ }^{106}$ lui confier la direction d'une école quasi universelle, et tant théorique, ${ }^{107}$ que pratique.

$\mathrm{Au}$ reste, il serait nécessair[e ${ }^{108}$ qu'un homme, qui jouit de toutes ces qualitées, fut encouragé à composer ${ }^{109}$ un livre élémentaire qui contint les principes ${ }^{110}$ de tout ce qui ${ }^{111}$ doit être enseigné d'après la $^{112}$ manière énoncée,

93 En interligne.

94 «les jeunes gens» remplace «ceux».

95 Remplace «incomplétement».

96 «sont destinés que pour» remplace «doivent».

97 «Comme les jeunes gens [...]» ajouté en marge.

98 En interligne: «de médecine».

99 Remplace un mot que je n'ai pu lire.

100 «de mieux les enchainer» remplace «de les réunir» suivi de deux ou trois mots que je n'ai pu lire.

101 Remplace «épargner».

102 Biffé: «et dès qu'il».

103 «naturellement chaqu'assertion» remplace «chaqu'objet».

104 Remplace «enseignés».

105 Biffé: «au lit des malades».

106 Biffé: «se fier au».

107 Surcharge «théorétique».

108 Remplace «à souhaiter».

109 En interligne: «à composer».

110 Remplace «élémens».

111 Remplace «qu'il» suivi d'un mot que je n'ai pu lire.

112 «d'après la» remplace «d'une». 
enfin $^{113}$ que ce livre put ${ }^{114}$ toujours servir de guide ${ }^{115}$ à ceux aux quels il aura été plus amplement ${ }^{116}$ expliqué.

Voilà, Monsieur le Comte, tout ce que j'ai pû Vous suggérer sur la demande dont V. E. m'a honnoré! Toujours intimement ${ }^{117}$ persuadé, que la Russie, pour que la santé de ses habitants soit bien soignée, au lieu de demi-médecins, ${ }^{118}$ a besoin de médecins parfaitement et bien ${ }^{119}$ instruits; et ${ }^{120}$ convaincu en même tems ${ }^{121}$ qu'on ne sauroit procurer à cet Empire ${ }^{122}$ des sujets disposés pour l'étude ${ }^{123}$ régulière en médecine, à moins que de les trouver ${ }^{124}$ dans la classe des prêtres: je reviens toujours au principe çi dessus exposé: qu'il faudroit réunir de nouveaux, comme cela s'est pratiqué chés tous les grands peuples de l'Antiquité, et ${ }^{125}$ dans les prémiers siècles chrétiens, l'état du prêtre à celui du médecin, ${ }^{126}$ pour le meilleur ${ }^{127}$ service du peuple de la campagne dans ses maladies. ${ }^{128}$

En soumettant ces idées au jugement profond de Votre Excellence, j'ai l'honneur d'être ${ }^{129}$ avec le plus grand respect Monsieur le Comte

Votre très humble et

très obéissant serviteur

jean pierre frank.

St Petersbourg le 12 février 1806.

\section{Bibliographie}

Ackerknecht, Erwin Heinz, La Médecine hospitalière à Paris (1794-1848) (Paris 1986) Annales littéraires et morales (Paris 1804)

Bonamour, Jean, A. S. Griboedov et la vie littéraire de son temps (Paris 1965)

Brambilla, Giovanni Ales., Discours sur la prééminence et l'utilité de la chirurgie (Bruxelles 1787)

Ceillier, Rémy, Histoire générale des auteurs sacrés et ecclesiastiques (Paris 1748)

113 Remplace «puis».

114 Remplace «puisse».

115 Biffé: «aux jeunes personnes».

116 «plus amplement» ajouté en marge.

117 En interligne.

118 «au lieu de demi-médecins,» ajouté en marge.

119 En interligne.

120 Remplace «mais»; biffé: «toujours».

121 En interligne: «en même tems».

$122 \mathrm{Il}$ avait d'abord écrit: «ces derniers à moins que d'y recourir aux prêtres» suivi de deux ou trois mots que je n'ai pu lire; puis remplacé par «cette classe de médecins», biffé pour donner la dernière leçon en interligne.

123 Biffé: un ou deux mots que je n'ai pu lire.

124 Biffé: «dans celle des prêtres».

125 «comme cela s'est pratiqué [...]» ajouté en marge et en interligne.

126 Biffé: «au moins».

127 En interligne.

128 Biffé: «Daignés recevoir».

129 En interligne. 
Charas, Moïse, Pharmacopée Royale Galénique et chymique (Paris 1676)

Corlieu, Auguste, Centenaire de la faculté de médecine de Paris (1794-1894) (Paris 1896)

Coury, Charles, L'Enseignement de la médecine en France, des origines à nos jours (Paris 1968)

Debono, Ludovic, La Médecine en Russie de 1801 à 1917, thèse de médecine, Université de Franche-Comté (Besançon 1997)

Les Remèdes charitables de Madame Fouquet, pour guerir a peu de frais toutes sortes de maux internes qu'externes [...] (Lyon 1685)

Frank, Johann Peter, System einer vollständigen medicinischen Polizey (Vienne 1817)

Frioux, Stéphane/Patrick Fournier/Sophie Chauveau, Hygiène et santé en Europe de la fin du XVIII e siècle aux lendemains de la première Guerre Mondiale (Paris 2011)

Goubina, Maria, «Quelques aspects de l'essor du libéralisme en Russie au début du XIX ${ }^{\mathrm{e}}$ siècle», dans Sylvie Martin (éd.), Circulation des concepts entre Occident et Russie (Lyon 2008)

Heller, Robert, "'Priest-doctors' as a rural health service in the age of enlightenment”, Medical History 20 (1976)

Huguet, Françoise, Les Professeurs de la faculté de médecine de Paris. Dictionnaire prosopographique, 1794-1939 (Paris 1991)

Lafont, Olivier, «Médicaments des villes, médicaments des champs, réglementation stricte, contre pragmatisme», Revue d'Histoire de la Pharmacie 334 (2002) 211-220

Lemery, Nicolas, Pharmacopée universelle (Paris 1698)

Montrond, Maxime de, Les médecins les plus célèbres (Lille 1852)

Mémoires biographiques de Jean-Pierre Frank et de Joseph Frank, son fils, rédigés par ce dernier, édités par Caroline Paliulis, Bibliothèque de l'Université de Vilnius, département des manuscrits, cote F21 (à paraître)

Migne, Jacques Paul, Encyclopédie théologique (Paris 1859)

Rohlfs, Heinrich, Geschichte der deutschen Medicin (Stuttgart 1880)

Taschenbuch der Wiener Universität für das Jahr 1804 (Vienne 1804)

Tissot, Samuel-Auguste-David, Avis au peuple sur sa santé (Lausanne 1761)

Trépardoux, Francis, «Les médicaments de la Cour, chronologie, mise en œuvre et résultats humanitaires», Revue d'Histoire de la Pharmacie 312 (1996) 374-377 